\title{
Nucleation and Growth Visualization of Self-Assembled Polymeric Micelles/Vesicles Using in Situ Liquid Cell-TEM
}

\author{
Vahid Jabbari, David J. Banner, Abhijit H. Phakatkar and Reza Shahbazian-Yassar \\ University of Illinois at Chicago, Chicago, Illinois, United States
}

Aqueous self-assembly of amphiphilic phospholipids and formation of cell membranes are vital for all living systems. Amphiphilic block copolymers (polymers with both hydrophilic and hydrophobic blocks or segments) and in a process so-called molecular self-assembly in a selective liquid medium, e.g. water, can assemble into varied intricate and biomimetic structures including spherical micelles, cylindrical or worm-shape micelles, vesicles with sizes ranging from nano- to micro-meter. In fact, these block copolymers are very similar to amphiphilic cell membrane forming phospholipids which consist of a hydrophilic head and a hydrophobic fatty acid tails. However, compared to the small molecule counterparts, self-assembled polymeric nanomaterials offer higher stability (slower exchange kinetics of polymer chains compared to small amphiphilic molecules enabling nonergodic or kinetically frozen systems) and durability (higher molecular length and chain entanglement leads to a thicker as well as more resilient vesicular membranes) which make them a strong candidate for applications in therapeutics and drug delivery, nanomaterial synthesis, nanoscale patterning, and catalysis [1-3].

Due to importance of fundamentals of formation and functional characteristic of complex synthetic and biological materials, it is vital to study mechanism of nucleation and growth of organic and polymer soft matter in solution. Recently, some research groups have successfully utilized transmission electron microscopy (TEM) to visualize structure of ex-situ self-assembled block copolymers. While visualization offered by TEM offers valuable insights toward structure and morphology of the self-assembled copolymers, yet, none of these techniques enable operando or real-time study of nucleation and growth of polymer self-assemblies (individual stages of core-corona formation) to gain temporal and spatial insight at nanoscale level and the individual stages are less clear and largely unknown. Furthermore, realtime visualization of self-assembly of block copolymers offer examination of established theories of polymer aggregation as well as reorganization and morphological evolution which expand our knowledge in this area. To best of our knowledge, there is only a single report on real-time visualization of block polymer self-assembly during in-situ polymerization. To achieve operando visualization, in 2018, Touve et al., have studied PISA-based formation of spherical micelles from block copolymers using in-situ liquid cell transmission electron microscopy (LC-TEM) where electron beam was used to initiate polymerization [3].

Due to a large gap existing between the current knowledge and detailed mechanism of nucleation and growth of various block copolymers in different liquid mediums and structure of self-assembled copolymers, we aim to study nucleation and growth of polymers formed by PISA in a real-time by in-situ graphene liquid cell-transmission electron microscopy (GLC-TEM) and other in-situ LC-TEM techniques where electron beam is also used to initiate the polymerization and the self-assembled polymer nanostructures are directly visualized during their formation and evolution. Graphene is impermeable to small molecules and have an excellent thermal and electrical conductivity which minimize damages from electron radiation such as charging, degradation, and ionization [4]. In this regard, PolyGMA-co-PolyB di-block copolymer where PolyGMA is water soluble and PolyB is water insoluble is synthesized in two steps (as shown in Fig. 1): (i) RAFT polymerization of glycerol methacrylate (GMA), (ii) using the resulting macromolecule PolyGMA, called macro-CTA or iniferter (a chemical compound which acts 
simultaneously as initiator, transfer agent, and terminator) to both initiate the polymerization of monomer $\mathrm{B}$ and form the second block and also control the chain growth through CTA. As it can be seen from the ${ }^{1}$ H NMR results in Fig. 1, GMA monomer polymerize over time to form PolyGMA block of the di-block copolymer and the rate of polymerization with respect to time is linear which can be indicative of a controlled/living radical polymerization. For the next step, monomer B is polymerized in the presence of the macro-CTA under and electron beam to form the target di-block PolyGMA-co-PolyB copolymer and hence, micelle/vesicle structure. The UV light is also used instead of electron beam to synthesize control samples for comparison. According to in-situ GLC-TEM shown in Fig. 2, polymerization of monomer B is successfully achieved and the resulting PolyGMA-co-PolyB di-block copolymer show spherical micelles (bright spheres shown by arrows) structure in water. Dark spots are related to the commercial $\mathrm{Cu}$ grid and are possibly due to formation of $\mathrm{Cu}$ particles after etching $\mathrm{Cu}$ grid in the process of graphene coating. Moreover, we have observed self-assembly of PolyGMA-co-PolyB di-block copolymer over $\mathrm{Cu}$ particles as shown in Fig. 2. Copolymers are assumed to be coated from their hydrophobic part onto hydrophobic $\mathrm{Cu}$ particles and encapsulated the particles, where similar observation for encapsulation of Au nanoparticles by micelles is reported elsewhere [1]. We study mechanism of nucleation and growth of the micelle/vesicle structures through changing the parameters such as composition of the copolymer, concentration of the copolymer in solution, nature and dosage of solvent, the presence external stimuli such as temperature, and electron beam dosage. Particularly, these parameters determine the aggregate morphology based on influencing the polymer blocks in the core, repulsion between the corona chains, and surface tension between the solvent and the core.
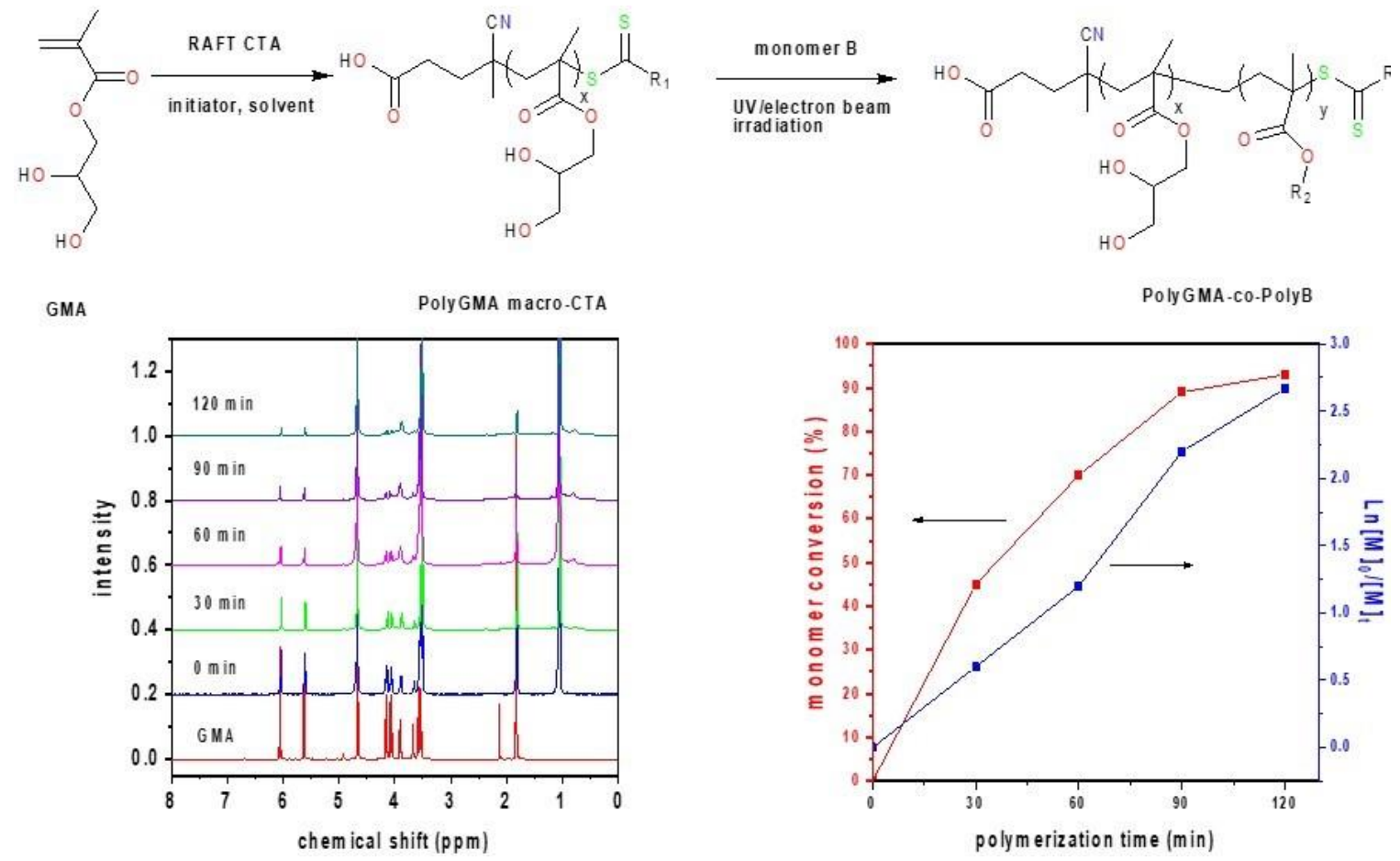

Figure 1. Chemical structure and synthetic protocol of the block copolymer, 1H NMR of GMA monomer and kinetics of RAFT polymerization of GMA measured by 1H NMR. 

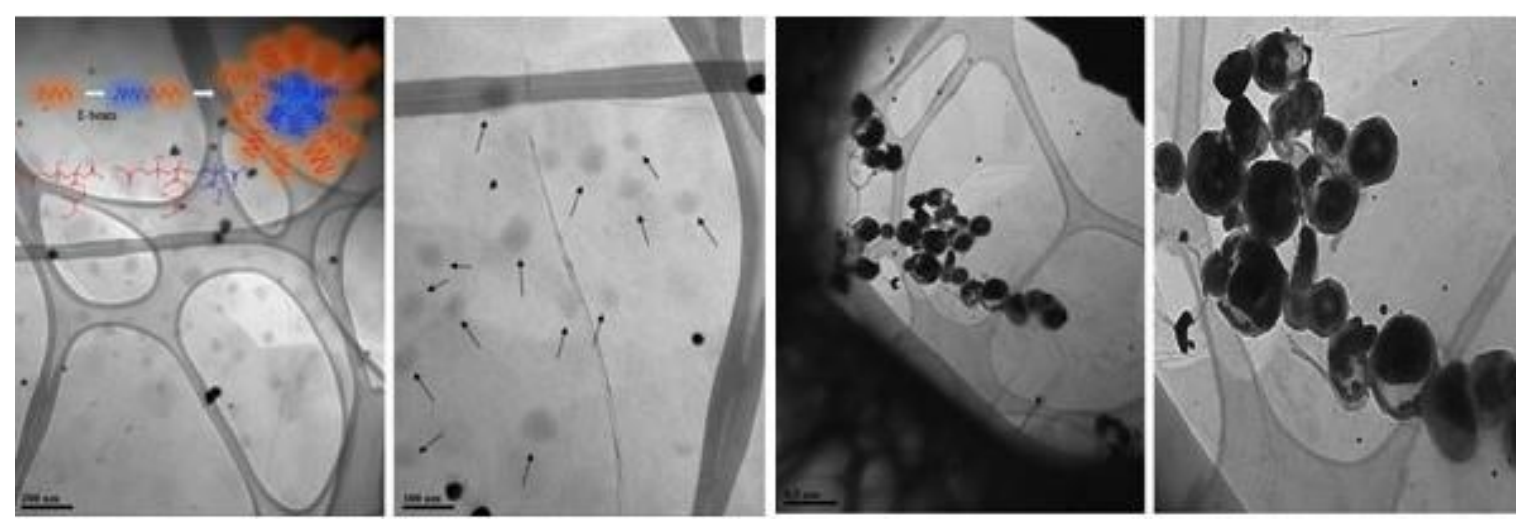

Figure 2. TEM images of free PolyGMA-co-PolyB spherical micelles (arrows show micelles and dark spots are exisiting $\mathrm{Cu}$ particles on the commerical grid after etching) formed after PISA under electrn beam and encapsulation of $\mathrm{Cu}$ nanoparticles by PolyGMA-co-PolyB micelles.

\section{References}

[1] Chang Li et al., Dynamics of amphiphilic block copolymers in an aqueous solution: direct imaging of micelle formation and nanoparticle encapsulation, Nanoscale, 2019, 11, 2299.

[2] Mollie A. Touve et al., Block Copolymer Amphiphile Phase Diagrams by High-Throughput Transmission Electron Microscopy, Macromolecules 2019, 52, 5529.

[3] Mollie A. Touve et al., Polymerization-Induced Self-Assembly of Micelles Observed by Liquid Cell Transmission Electron Microscopy, ACS Cent. Sci. 2018, 4, 543.

[4] Kandula Hima Nagamanasa et al., Liquid-Cell Electron Microscopy of Adsorbed Polymers, Adv. Mater. 2017, 29, 1703555. 